\title{
Laparoendoscopic single-site nephroureterectomy for morbid obese patients
}

Cesar Augusto Braz Juliano, Alexandre Stievano Carlos, Renato Meirelles Mariano Costa Junior, Marcos Tobias-Machado, Antonio Carlos Lima Pompeo

Department of Urology, ABC Medical School (CABJ, RMMCjr, ACLP, MTM), Santo André and Hospital Santa Marcelina (ASC), Sao Paulo, Brazil

\section{ABSTRACT}

Since the first laparoendoscopic single-site (LESS) surgery report in urology in 2007 (1) (Rane A e Cadeddu JA), the few reports of LESS extraperitoneal access in the literature were mainly described for less complex cases. The aim of this video is to demonstrate the feasibility of LESS extraperitoneal access in a morbid obese patient presenting a malignant tumor in the renal pelvis.

The patient is positioned in 90-degree lateral decubitus. An incision is made below the abdominal skin crease on the left side of the patient and the anterior rectus fascia is vertically incised with manual dissection of the extra/retroperitoneal space. We use an Alexis ${ }^{\circledR}$ retractor to retract the skin maximizing the incision orifice. Three trocars $(12,10$ and $5 \mathrm{~mm}$ ) are inserted through a sigle-port. The pedicle was controlled "en bloc" with a vascular stapler and the bladder cuff treated by the conventional open approach through the same incision.

Operative time was 126 minutes with minimal blood loss. The pathology reported high grade papillary urothelial carcinoma in the pelvis (pT3NOM0) and in the ureter (pTa).

LESS extraperitoneal nephroureterectomy is feasible and safe, even in more complex cases. It is a good alternative for morbid obese patients and for patients with synchronous distal ureteral tumors for whom an open approach to the bladder cuff is proposed to avoid incisions in two compartments of the abdominal wall.

\section{ARTICLE INFO}

Available at: www.brazjurol.com.br/videos/november_december_2013/Juliano_895_896video.htm

Int Braz J Urol. 2012; 39 (Video \#15): 895-8

Submitted for publication:

October 01,2013

Accepted after revision:

November 30, 2013

\author{
Correspondence address: \\ Cesar Augusto Braz Juliano, MD \\ Department of Urology - ABC Medical School \\ Rua Maestro Cardim, $1251 \mathrm{cj} .34$ \\ Sao Paulo, SP, 01323-000, Brazil \\ Fax: + 5511 3262-1575 \\ E-mail: drcesarjuliano@yahoo.com.br
}




\section{EDITORIAL COMMENT}

The authors demonstrate a very nice and useful approach for a retroperitoneal nephroureterectomy in an obese patient with left upper tract urothelial carcinoma. Furthermore, they reveal their ingenuity in utilizing readily available "home-made" materials to facilitate a LESS technique. Without doubt, the retroperitoneal approach has distinct advantages over transperitoneal access for certain clinical scenarios to include obesity - as evidenced in this video. Other advantages from a surgical prospective include the avoidance of a hostile abdomen in patients who have experienced prior extensive abdominal surgery and posteriorly situated tumors in patient candidates for minimally invasive retroperitoneal partial nephrectomies. Clinically, patients managed with minimally invasive retroperitoneal techniques often have less pain and abdominal ileus, and they frequently experience earlier hospital discharge and convalescence compared to transperitoneal laparoscopic approaches.

Lost in this particular video however, is the additional decision making that urologists should consider when managing patients with upper tract disease. In this particular instance, the patient had pre-operative radiographic evidence of locally advanced cancer, confirmed with the final pathology revealing multifocal, high-grade, invasive urothelial carcinoma (pathologic T3a tumor). While the role and the extent of lymphadenectomy for upper tract urothelial carcinoma has not been clearly elucidated (compared to cystectomy for bladder urothelial carcinoma), a formal lymphadenectomy should be considered under clinical circumstances as demonstrated in this patient example. Simply demonstrating another technical modification to removing a kidney, while worthwhile, may not be as important as defining improved measures to optimize rates of long-term cancer-specific and overall survival. Along this same pathway is the shifting paradigm towards utilizing neoadjuvant chemotherapy for possible locally advanced upper tract urothelial carcinoma. Many patient candidates for cisplatin-based neoadjuvant chemotherapy are rendered inappropriate candidates for cisplatin-based regimens postoperatively following nephroureterectomy due to the now compromised renal function as evidenced by diminished renal glomerular filtration and the potential for chemotherapy-induced renal toxicity. Careful integration of appropriate surgical volume and technique, chemotherapy, or other biologic modifiers will be required to make some impact towards improved oncologic outcomes in patients with advanced upper tract disease.

Wade J. Sexton, MD

Associate Member Urologic Oncology Fellowship Director Genitourinary Oncology Program Moffitt Cancer Center Tampa, Florida E-mail:wade.sexton@moffitt.org 